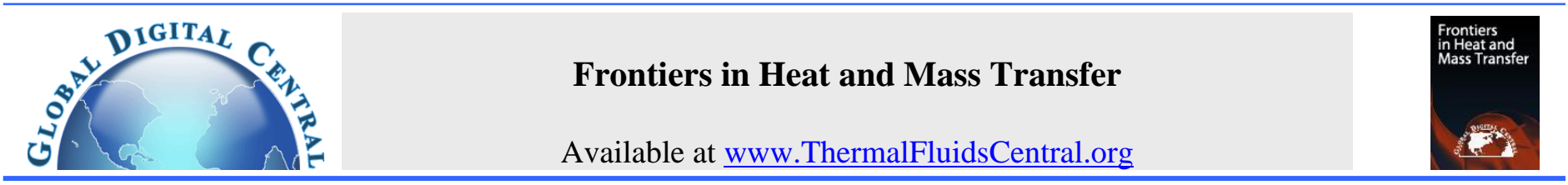

\title{
THE SIMULTANEOUS EFFECT OF ECKERT NUMBER AND MAGNETIC PRANDTL NUMBER ON CASSON FLUID FLOW
}

\author{
Silpisikha Goswami ${ }^{\mathrm{a}, \mathrm{b}}$ and Dipak Sarma ${ }^{\mathrm{b}}$ \\ ${ }^{a}$ Institute of Advanced Study in Science and Technology, Guwahati, Assam, 781035, India \\ ${ }^{\mathrm{b}}$ Cotton University, Guwahati, Assam, 781001, India
}

\begin{abstract}
This study includes the observation of electrically conducting non-Newtonian fluid flow through a vertical porous plate considering the effect of the induced magnetic field. Our approach is numerical to investigate how the variation of magnetic Prandtl number and Eckert number effect the flow profiles. Influence of Casson parameter and Hartmann number in the profiles is also observed and depicted in graphs. The rate of heat transfer, the rate of mass transfer and the skin friction are calculated and presented in tables. A significant effect of magnetic Prandtl number and Eckert number is observed. We compared our results with some previous analytical and numerical works.
\end{abstract}

Keywords: Casson parameter, Induced magnetic field, vertical porous plate, Finite Difference Method, electrically conducting fluid

\section{INTRODUCTION}

The study of the flow of Casson fluids in a porous medium has a significant influence on industrial, agricultural as well as medical research. Since the viscosity of the Casson fluid is dependable on the yield stress, various circumstances of blood flow can be studied with the help of Casson model. In agricultural research, the flow of the mixture of manure in the soil can be studied assuming it as the flow of Casson fluid model. Casson introduced this model in 1959 to describe a flow equation for pigment oil-suspensions of printing ink. Farther, this model has been studied by various researchers in theoretical as well as in experimental field to explore the different characteristics of shear-thinning fluid flow. Kenjeres (2008) contributed to the study for delivery of the drug in the affected area. (Mukhopadhyay et al., 2013) investigated the effect of Casson parameter on an unsteady flow over a stretching sheet. (Completo et al., 2014)investigated experimentally and theoretically the blood destructive phenomena and compared various Newtonian and non-Newtonian fluids. (Khalid et al., 2015) approached an analytical investigation to observe the Casson fluid flow past an oscillating vertical plate.

Casson nanofluid flow is another emerging area of fluid dynamics. (Sulochana et al., 2016) performed similarity solution compared the 3D MHD Newtonian and Casson Nano Fluid flow. (Ullah et al., 2017) investigated the effect of slip condition on a Casson fluid flow. (Siddiqa et al., 2018) investigated the Casson fluid flow in a wavy cone with the radiating surface. (Reddy et al., 2018a) observed the stable and convergent numerical results of Casson fluid flow through an oscillating sheet. (Hsua et al., 2019) investigated numerically the MHD flow of non-Newtonian fluid over a vertical plate embeded in a porous medium.

Most of the research of Casson fluid flow conducted by avoiding the effect of the induced magnetic field. Magnetic field plays an impor- tant rule as the controller of the rate of cooling of a machine and helps to obtain the required product. Moreover considering the presence of a magnetic field has a significant influence on MHD energy generator systems, thermo magneto aerodynamics and nuclear reactors. Ibrahim (2016) investigated the effect of the induced magnetic field on fluid flow in a stagnation point. (Hayat et al., 2018) observed the effect of melting heat transfer and the induced magnetic field in fluid flow. Arora and Gupta (1972) investigated the effect of the radial magnetic field on the magnetohydrodynamic flow between two rotating coaxial cylinders. M. Kumari and Nath (1990) studied the effect of the induced magnetic field on the heat transfer over a stretching field. (Mekheimer et al., 2012) approached analytically to observe the peristaltic flow of second-order fluid in the presence of an induced magnetic field in a channel. (Jafar et al., 2013) observed the MHD boundary layer flow in the presence of the induced magnetic field. (Raju et al., 2016) et al. investigated the effect of the induced magnetic field on the stagnation flow of a Casson fluid. (Animasaun et al., 2016) et al. approached analytically a parametric study of Casson fluid flow with variable thermo physics property. (Ahmed et al., 2017) investigated the squeezing Casson fluid flow between two parallel plates in the presence of a magnetic field. (Reddy et al., 2018a) investigated the Joule heating effect on Casson fluid. Alkasasbeh (2018) investigated numerically influence thermal radiation in MHD flow of micropolar Casson fluid flow in a horizontal cylindrical surface. Alkasasbeh (2017) investigated the influence of thermo diffusion and diffusion thermo effect on MHD heat flow of non-Newtonian fluid.

In this study, we observed the Casson fluid flow by considering the effect of the induced magnetic field. The assumed two-dimensional configuration is placed in a porous medium with the flow of viscous, elec-

${ }^{\dagger}$ Corresponding author. Email: gsilpisikha@gmail.com 
trically conducting fluid. The influence of involved physical parameters on the velocity, temperature, concentration, rate of heat transfer, rate of mass transfer and skin friction was depicted in graphs and tables. It is a extended work of (Reddy et al., 2018b) by introducing induced magnetic field to it. The results appeared to be great agreement with the previous works.

\section{MATHEMATICAL FORMULATION}

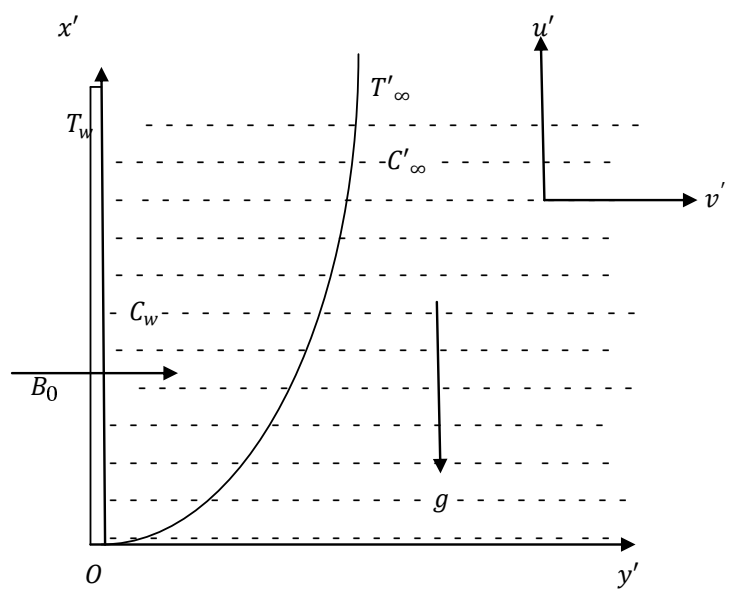

Fig. 1 Physical formulation of the problem

The physical transformation of the problem is assumed to be in a two-dimensional system with vertical porous plate through which an electrically conducting, incompressible, non-Newtonian and viscous fluid is passing with constant velocity along $y$ direction. The problem is visualised in a Cartesian coordinate system, where $x^{\prime}$ axis is considered along with the plate while $y^{\prime}$ is placed perpendicular to the plate. A constant magnetic field $B_{0}$ is applied normal to the plate. A comparatively high process is assumed under free convection.

The rheological equations are stated below,

$$
\tau_{i j}= \begin{cases}2\left(\frac{\mu_{B}+P_{y}}{\sqrt{2 \pi}} e_{i j}\right) & \text { if } \pi \geq \pi_{c} \\ 2\left(\frac{\mu_{B}+P_{y}}{\sqrt{2 \pi_{c}}} e_{i j}\right) & \text { if } \pi<\pi_{c}\end{cases}
$$

Here, $\pi=e_{i j} e_{i j}$, the product of component of deformation rates controls the flow.

As the plate grows infinitely in vertical direction, all the variable varies along $y^{*}$ axis only. therefore the continuity equation can be stated as,

$$
\frac{\partial v^{*}}{\partial y^{*}}=0
$$

$v=U$ is considered as the uniform velocity along $y$ axis. This nonNewtonian unsteady fluid flow is governed by the following equations, Momentum equation:

$$
\begin{aligned}
-\nu_{0} \frac{\partial u^{*}}{\partial t^{*}}= & \left(1+\frac{1}{\gamma}\right) \frac{\partial^{2} u^{*}}{\partial y^{*^{2}}}+g \beta\left(T^{*}-T_{\infty}^{*}\right)+g(\beta)^{*}\left(C^{*}-C_{\infty}^{*}\right)-\frac{\nu}{K^{*}} u^{*} \\
& +\frac{\mu_{c} H_{0}}{\rho} \frac{\partial H_{x}^{*}}{\partial y^{*}}
\end{aligned}
$$

Energy equation:

$$
\begin{aligned}
-\nu_{0} \frac{\partial T^{*}}{\partial t^{*}} & =\frac{\kappa}{\rho C_{p}} \frac{\partial^{2} T^{*}}{\partial y^{*^{2}}}-\frac{1}{\rho C_{p}} \frac{\partial q_{r}^{*}}{\partial y^{*}}+\frac{Q_{0}}{\rho C_{p}}\left(T^{*}-T_{\infty}^{*}\right)+\frac{D_{m} k_{T}}{C_{s} C p} \frac{\partial^{2} C^{*}}{\partial y^{*^{2}}} \\
& +\frac{1}{\sigma \rho C_{p}}\left(\frac{\partial H_{x}^{*}}{\partial y^{*}}\right)^{2}
\end{aligned}
$$

Concentration equation:

$$
-\nu 0 \frac{\partial C^{*}}{\partial t^{*}}=D_{M} \frac{\partial^{2} C^{*}}{\partial y^{*^{2}}}-K_{r}^{*}\left(C^{*}-C_{\infty}^{*}\right)+\frac{D_{T} K_{t}}{T_{m}} \frac{\partial^{2} T^{*}}{\partial y^{*^{2}}}
$$

Magnetic induction equation:

$$
-v_{0} \frac{\partial H_{x}^{*}}{\partial y^{*}}=H_{0} \frac{\partial u^{*}}{\partial y^{*}}+\frac{1}{\sigma \mu_{c}} \frac{\partial^{2} H_{x}^{*}}{\partial y^{*^{2}}}
$$

Following are the required initial and boundary conditions for the respective flow profiles,

$$
\begin{gathered}
u^{*}=0, T^{*}=0, C^{*}=0, H^{*}=0 \forall y^{*} \text { and } t^{*} \leq 0 \\
u^{*}=U, T^{*}=T_{w}+\epsilon\left(T_{w}^{*}-T_{\infty}^{*}\right) e^{n^{*} t^{*}}, C^{*}=C_{w}^{*}+\epsilon\left(C_{w}^{*}-C_{\infty}^{*}\right), \\
\left(H_{x}\right)^{*}=\left(H_{w}\right)^{*} \text { at } y^{*}=0 \\
u^{*} \rightarrow 0, T^{*} \rightarrow T_{\infty}^{*}, C^{*} \rightarrow C_{\infty}^{*},\left(h_{x}\right)^{*} \longrightarrow 0 \text { as } y^{*} \rightarrow \infty
\end{gathered}
$$

The thermal radiation heat flux gradient can be assumed as, $\frac{\partial q_{r}}{\partial y^{*}}=4 a^{*} \sigma^{*}\left(T_{\infty}^{*}-T^{*^{4}}\right)$

We assume the negligible temperature difference to get $T^{*}$ as a linear function. Neglecting the higher orders of the Taylor's series we get, $T^{*^{4}} \cong 4 T^{*^{3}} T^{*}-3 T_{\infty}^{* 4}$

With the help of following non-dimensional quantities we convert the above partial differential equations into non-dimensional form,

$$
\begin{gathered}
u=\frac{u^{*}}{U}, y=\frac{U y^{*}}{\nu}, t=\frac{U t^{*}}{\nu}, \theta=\frac{T^{*}-T_{\infty}^{*}}{T_{w}^{*}-T_{\infty}^{*}}, \phi=\frac{C^{*}-C_{\infty}^{*}}{C_{w}^{*}-C_{\infty}^{*}}, \\
Q=\frac{Q_{0} \nu}{\rho C_{p} U^{2}}, K=\frac{K^{*} u_{0}^{2}}{\nu^{2}}, \operatorname{Pr}=\frac{\mu C_{p}}{k}, H=\sqrt{\frac{\mu_{c}}{\rho} \frac{H_{x}^{*}}{U}}, \\
G r=\frac{\nu \beta g\left(T_{w}^{*}-T_{\infty}^{*}\right)}{U^{2}}, G_{c}=\frac{\nu \beta^{*} g\left(T_{w}^{*}-T_{\infty}^{*}\right.}{U^{3}}, K r=\frac{K_{r}^{*} \nu}{U^{2}}, S c=\frac{\nu}{D}, \\
R=\frac{16 a^{*} \nu \sigma^{*} T_{\infty}^{* 3}}{\rho C_{p} U^{2}}, D u=\frac{D_{m} k_{T}\left(C_{w}^{*}-C_{\infty}^{*}\right.}{\nu C_{s} C_{p}\left(T_{w}^{w}-T_{\infty}^{*}\right)}, \mu=\nu \rho, \\
S r=\frac{D_{T} K_{T}\left(T_{w}^{*}-T_{\infty}^{*}\right)}{\nu T_{M}\left(C_{w}^{*}-C_{\infty}^{*}\right)}, E c=\frac{U^{2}}{C_{p}\left(T_{w}^{*}-T_{\infty}^{*}\right)}
\end{gathered}
$$
low,

The non-dimensional form of the governing equations are given be-

$$
\begin{gathered}
\frac{\partial u}{\partial t}=\left(1+\frac{1}{\gamma}\right) \frac{\partial^{2} u}{\partial y^{2}}+G r \theta+G m \phi-\frac{u}{k}+M \frac{\partial H}{\partial y} \\
\frac{\partial \theta}{\partial t}=\frac{1}{P r} \frac{\partial^{2} \theta}{y^{2}}-(R-Q) \theta+D u \frac{\partial^{2} \phi}{\partial y^{2}}+\operatorname{PmEc}\left(\frac{\partial H}{\partial y}\right)^{2} \\
\frac{\partial \phi}{\partial t}=\frac{1}{S c} \frac{\partial^{2} \phi}{\partial y^{2}}-K r \theta+S r \frac{\partial^{2} \theta}{\partial y^{2}} \\
\frac{\partial H}{\partial t}-\frac{\partial H}{\partial y}=M \frac{\partial u}{\partial y}+\frac{1}{P m} \frac{\partial^{2} H}{\partial y^{2}}
\end{gathered}
$$

Non-dimensional boundary conditions are,

$$
\begin{gathered}
u=1, \theta=1+\epsilon e^{n t}, \phi=1+\epsilon e^{n t}, H=h \text { at } y=0 \\
u \rightarrow 0, \theta \rightarrow 0, \phi \rightarrow 0, H \rightarrow 0 \text { as } y \rightarrow \infty
\end{gathered}
$$

Our model has been configured with this set of partial differential equations and boundary conditions. 


\section{SOLUTION OF THE PROBLEM}

Our approach is numerical to obtain the fluid profiles from the above set of equations. Finite Difference Method(FDM) is one of the most appropriate method for unsteady flow to get the approximate results. The used FDM technique is given below,

$$
\begin{gathered}
\frac{\partial Z}{\partial t}=\frac{Z(i+1, j)-Z(i, j)}{\Delta t} \\
\frac{\partial Z}{\partial y}=\frac{Z(i, j+1)-Z(i, j)}{\Delta t} \\
\frac{\partial^{2} u}{\partial y^{2}}=\frac{Z(i, j+1)-2 Z(i, j)+Z(i, j-1)}{\Delta y^{2}}
\end{gathered}
$$

Where, $Z$ represents the non-dimensional flow characteristic. Using the above technique we convert the set of PDE to a set of algebraic equations. These equations were solved in MATLAB to get the required results.

\section{RESULTS AND DISCUSSION}

We observe the simultaneous effect of magnetic Prandtl number and Eckert number in the fluid profiles. The high value of both the parameters leads to a zigzag motion in the temperature profile far away from the plate. For large Ec.Pr energy dissipation is getting charged actively for which the influence of the kinetic energy plays the determining role in temperature distribution and heat transfer. For higher value $E c$, also the effect of suction occurs.

For physical relevance of the problem, we consider the $\operatorname{Pr}$ value within 0.7-1 which corresponds to air. The value of $S c$ is considered as 0.22 which corresponds to Hydrogen in $25^{\circ} \mathrm{C}$ and 1 atmospheric pressure.

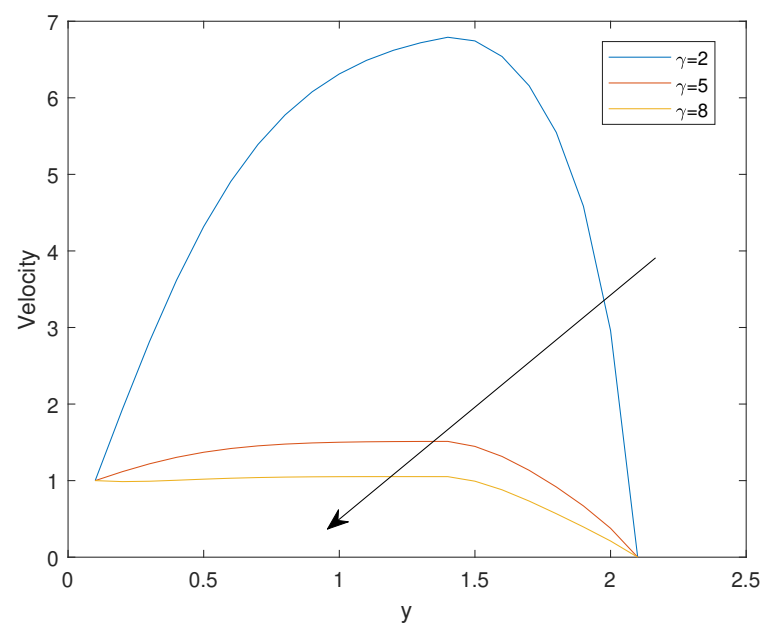

Fig. 2 Change in $u$ for various $\gamma$

Fig. 1-4 depict the changes in velocity, induced magnetic field, temperature and concentration profiles respectively concerning Casson parameter. Increasing Casson parameter decelerates velocity. Increase in non-Newtonian Casson parameter decreases yield stress which leads to an increase in plastic viscosity. This phenomenon resists fluid flow. Increasing $\gamma$ strengthens induced magnetic field vector. Increase in Casson parameter leads to increase in viscosity of the fluid due to which the fluid getting steadier that can justify the increase in induced magnetic field. The temperature profile increases with increasing $\gamma$. Increasing $\gamma$ leads to enhancing viscocity of the fluid which imply the increase in temperature. At the free stream concentration increases with increasing $\gamma$. Increase in viscosity makes the fluid thicker which leads to increase

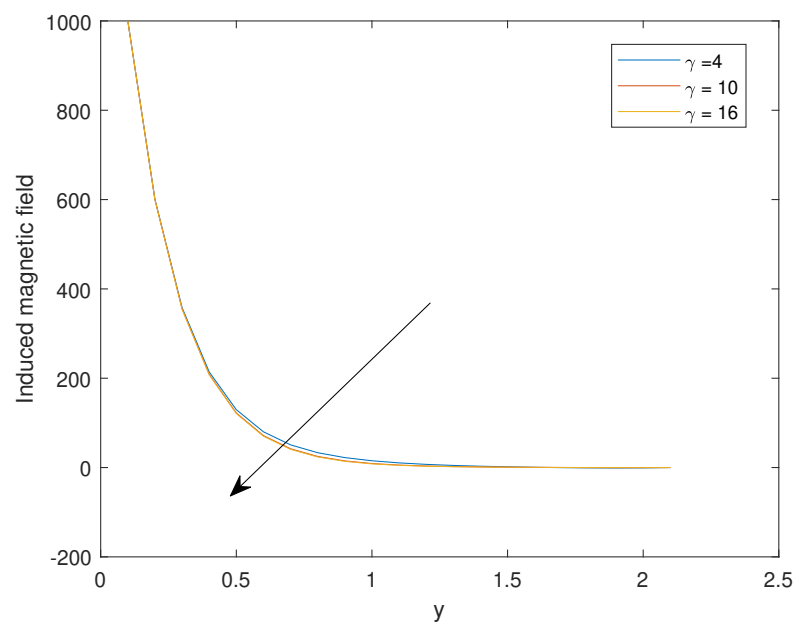

Fig. 3 Change in $H$ for various $\gamma$

Table 1 Change in $C f, N u$ and $S h$ for various $\gamma$

\begin{tabular}{|c|c|c|c|}
\hline$\gamma$ & Skin friction & Nusselt number & Sherwood number \\
\hline 4 & 6.662831 & 5.547007 & 1.762859 \\
\hline 10 & -2.257341 & 5.535174 & 1.765259 \\
\hline 16 & -3.694761 & 5.533758 & 1.765546 \\
\hline
\end{tabular}

in concentration. The behavioural change of velocity and temperature profiles can be related to the previous work of Mukhapadhyay (2013).

Table 1 depicts the changes in skin friction, Nusselt number and Sherwood number for the various values of $\gamma$. The changes appeared to be in good agreement with the previous work of (Shateyi et al., 2017), (Hayat et al., 2012) and Mukhapadhyay (2013).

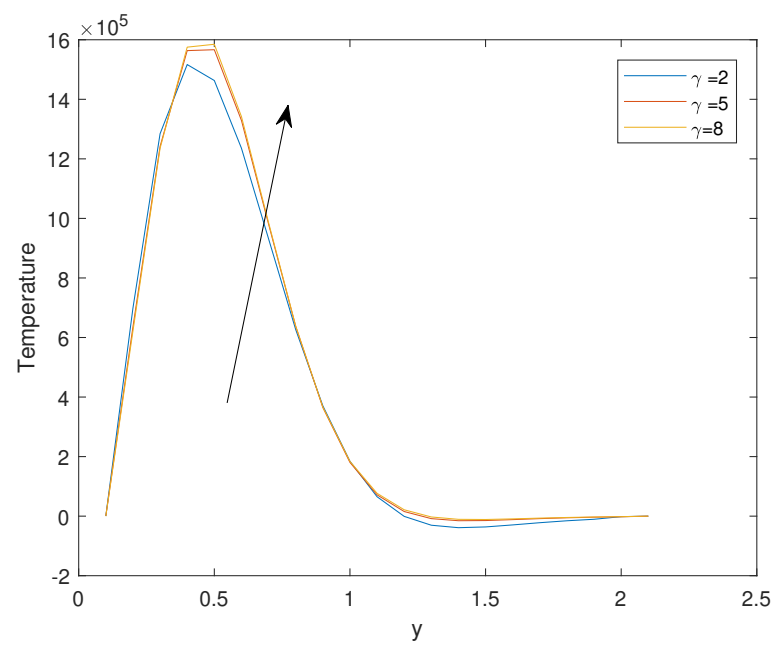

Fig. 4 Change in $\theta$ for various $\gamma$

Fig. 5-7 replicate the influence of Eckert number in the flow profiles. Velocity increases with increasing $E c$. Increased $E c$ implies increased in kinetic energy of the flow which leads to an increase in velocity. Induced magnetic field decreases with an increasing value of $E c$. By controlling the magnetic Prandtl number to a diminutive value, we observe an increasing $\theta$ far away from the plate with increasing $E c$. Ec can be expressed as the ratio of the dynamic temperature to the temperature, i. e. accelerating $E c$ can lead to an increase in dynamic temperature, which can explain the increasing phenomena of $\theta$. 


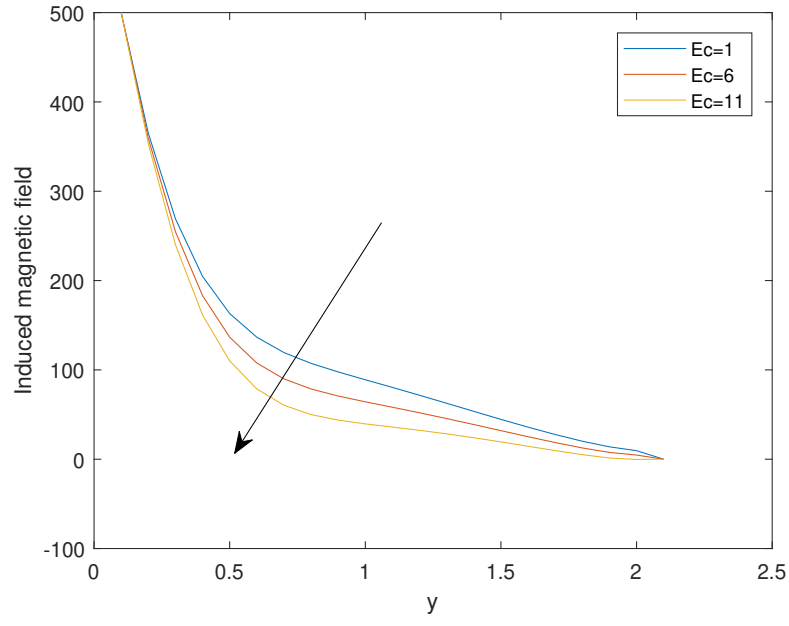

Fig. 5 Change in $H$ for various $E c$

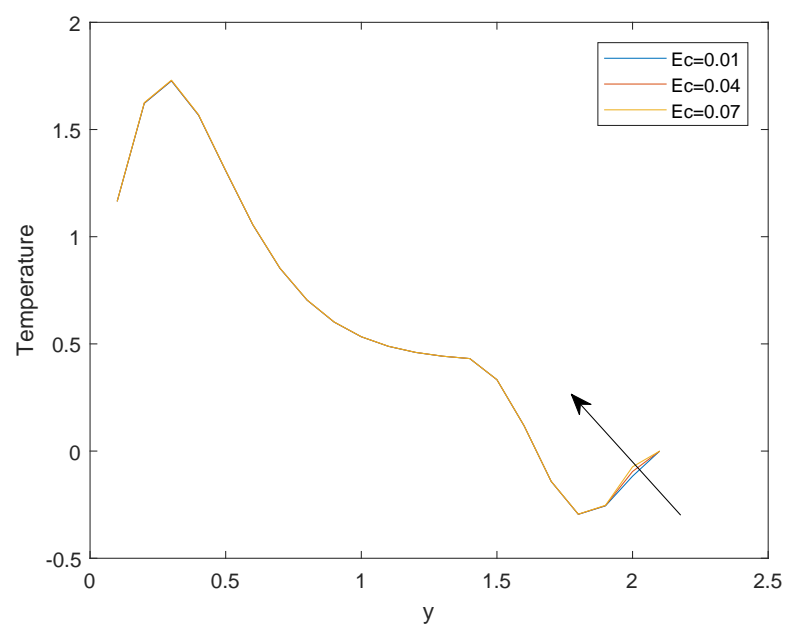

Fig. 6 Change in $\theta$ for various $E c$

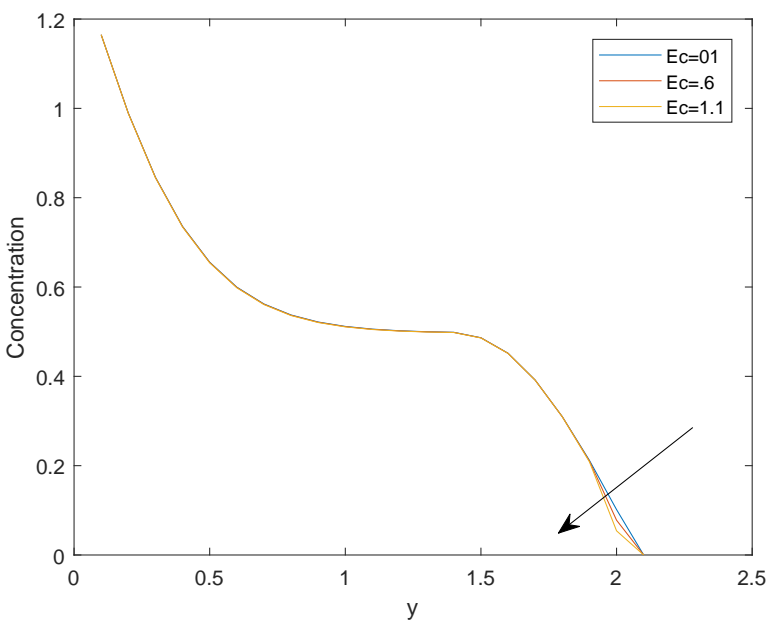

Fig. 7 Change in $\phi$ for various $E c$
Table 2 indicates the changes in skin friction, Nusselt number and Sherwood number for the various values of $E c$. It is observed that the direction of changes agrees with Chamkha (2004).

Table 2 Change in $C f, N u$ and $S h$ for various $E c$

\begin{tabular}{|c|c|c|c|}
\hline$E c$ & Skin friction & Nusselt number & Sherwood number \\
\hline 0.01 & 40.103803 & 5.269703 & 2.046907 \\
\hline 0.04 & 40.106399 & 5.418092 & 2.040367 \\
\hline 0.07 & 40.108996 & 5.566493 & 2.033827 \\
\hline
\end{tabular}

Fig.8-12 show the variations in the fluid profiles according to the increasing value of magnetic Prandtl number. With the increasing value of $P m$ velocity decreases. $P m$ is the ratio of viscous diffusion rate to the magnetic diffusion rate. i. e. increase in $\mathrm{Pm}$ can increase the viscous diffusion rate, which can be a cause of the decrease in velocity. Induced magnetic field vector decreases with increasing $P m$. Whereas, the temperature is decreasing and concentration is increasing for increasing $E c$. Increase of viscous diffusion rate for an increase in $\mathrm{Pm}$ can be a reason for these phenomena.

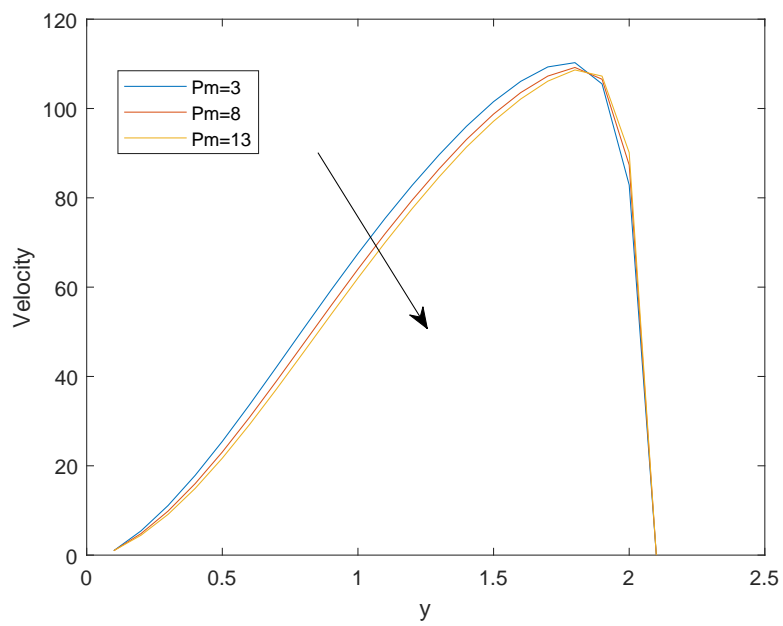

Fig. 8 Change in $u$ for various $P m$

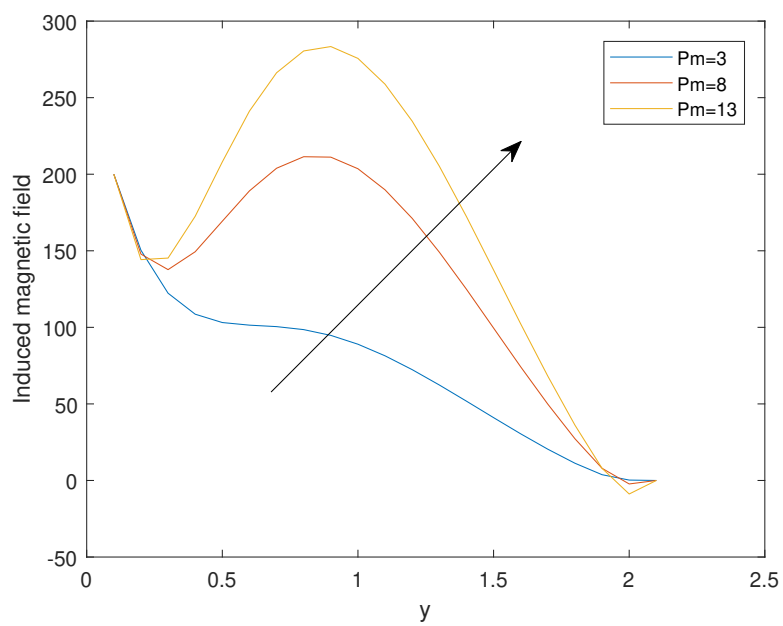

Fig. 9 Change in $H$ for various $\mathrm{Pm}$

Table 3 depicts the changes of Skin friction, Nusselt number and Sherwood number for various values of $\mathrm{Pm}$. 


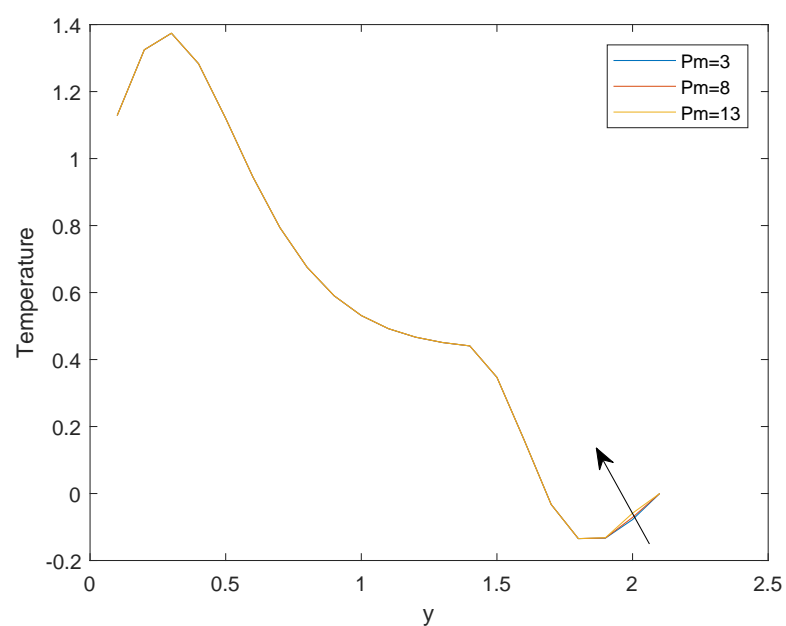

Fig. 10 Change in $\theta$ for various $P m$

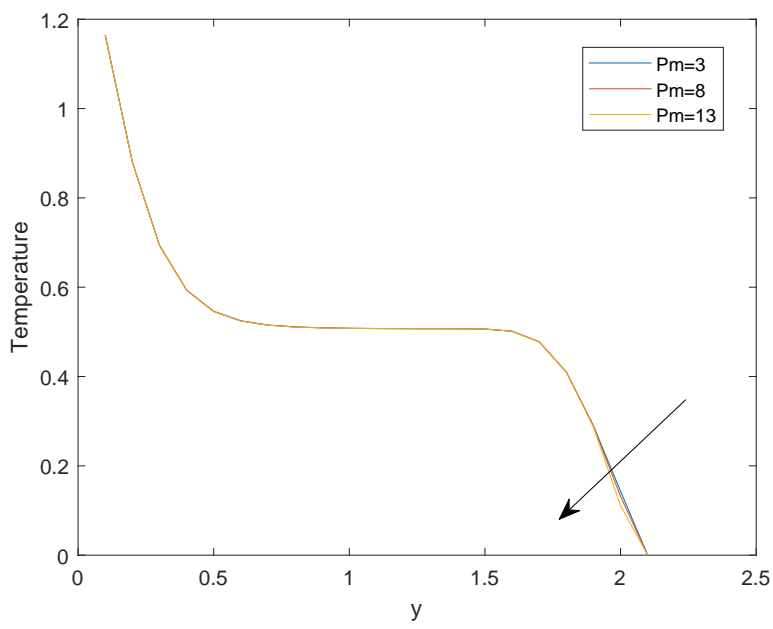

Fig. 11 Change in $\phi$ for various $P m$

Table 3 Change in $C f, N u$ and $S h$ for various $P m$

\begin{tabular}{|c|c|c|c|}
\hline$P m$ & Skin friktion & Nusselt number & Sherwood number \\
\hline 1 & 38.851924 & 7.505373 & 1.602364 \\
\hline 3 & 30.573980 & 80.127925 & 80.127925 \\
\hline 5 & 25.845169 & 266.282747 & -34.964690 \\
\hline
\end{tabular}

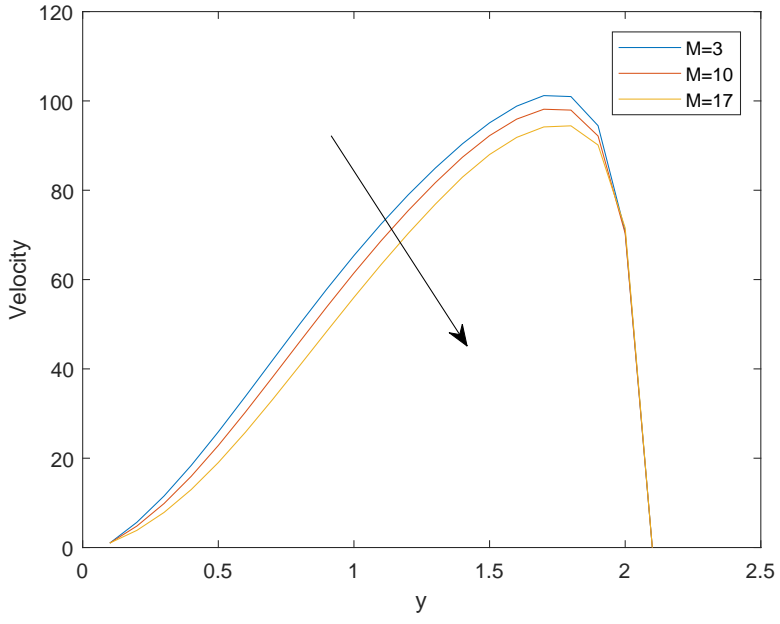

Fig. 12 Change in $u$ for various $M$

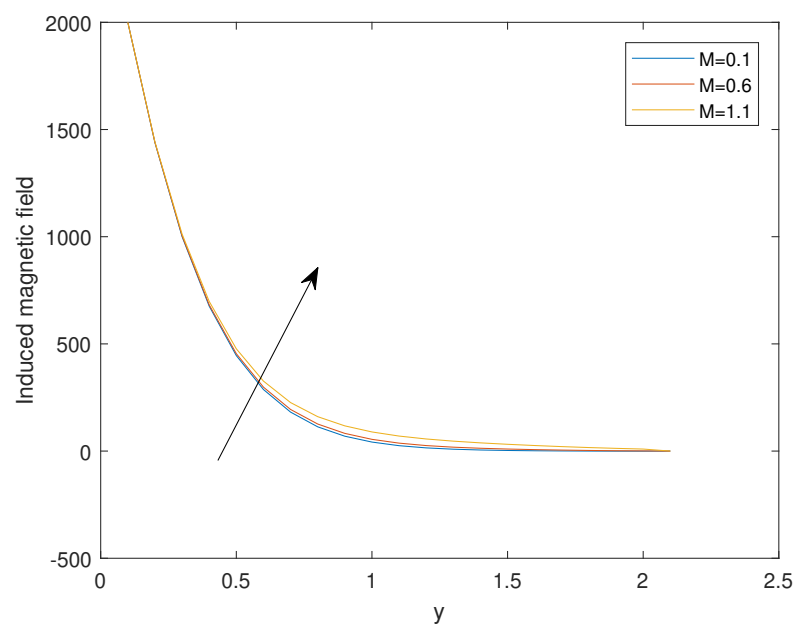

Fig. 13 Change in $H$ for various $M$

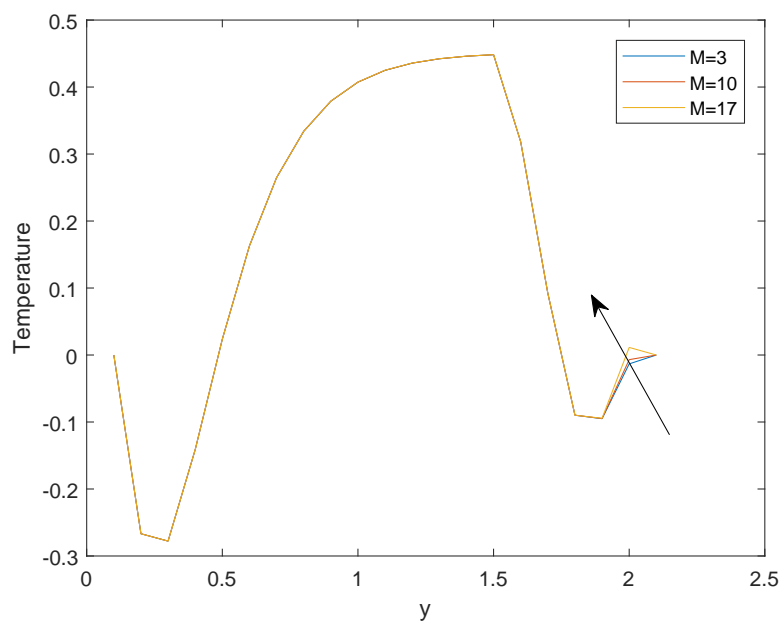

Fig. 14 Change in $\theta$ for various $M$ 


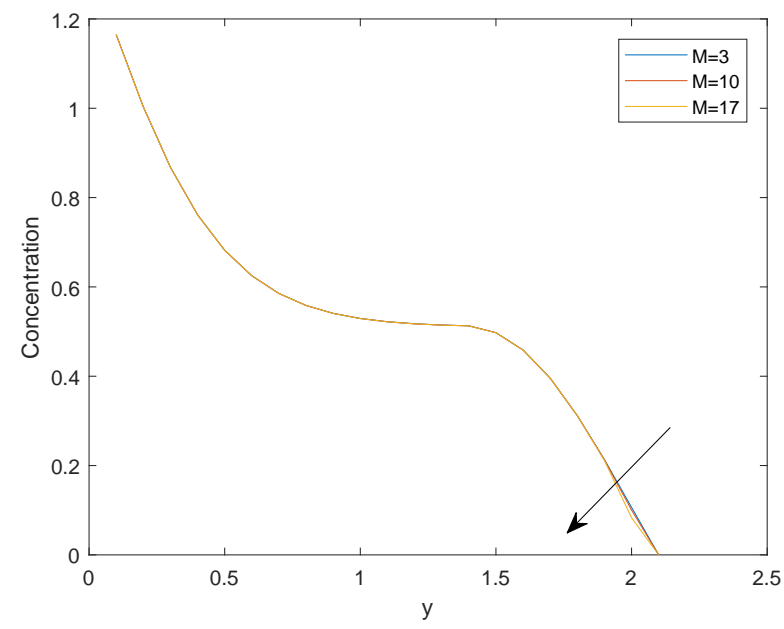

Fig. 15 Change in $p h i$ for various $M$

Fig. 13-16 explain the changes in the fluid profiles concerning the variation of Hartman number. Since an increase in Hartman number produces Lorenz force which is responsible for reducing the velocity as shown in figure 13. By controlling both $E c$ and $P m$ to a constant opposing value we finally got an increasing $\theta$ far away from the plate with an increasing value of $M$. Whereas, concentration decreases with increasing $M$.

Table 4 Change in $C_{f}, N u$ and $S h$ for various $M$

\begin{tabular}{|c|c|c|c|}
\hline$M$ & Skin friction & Nusselt number & Sherwood number \\
\hline 0.2 & 46.488641 & 2.248398 & 2.213756 \\
\hline 0.5 & 46.218368 & 2.248594 & 2.213747 \\
\hline 1.2 & 45.823684 & 2.248874 & 2.213735 \\
\hline
\end{tabular}

Table 4 depicts the changes of Skin friction, Nusselt number and Sherwood number for various values of $M$.

\section{CONCLUSION}

Our foremost motive was to observe the controlling power of $E c$ and $P m$ on characteristic profiles. We can conclude by justifying the established result that $E c$ and $P m$ has a stable influence specifically on the temperature profile if both are inversely proportionate, e.i. Ec.Pm = constant. Casson fluid flow in a porous medium has its influence on many industrial purposes. Further, this problem can be investigated by observing the effect of chemical reaction parameter and radiation.

\section{ACKNOWLEDGEMENTS}

The authors would like to thank the reviewers for their valuable suggestions regarding this study.

\section{NOMENCLATURE}

$B_{0} \quad$ magnetic field(Tesla)

$C \quad$ fluid concentration

$C_{\infty} \quad$ free stream concentration

$C_{p} \quad$ specific heat capacity at constant pressure $(\mathrm{J} / \mathrm{kg} \cdot \mathrm{K})$

$D_{M} \quad$ molecular diffusivity $\left(\mathrm{m}^{2} / \mathrm{s}\right)$

$D_{M} \quad$ coefficient of heat diffusivity()

$D_{T} \quad$ coefficient of mass diffusivity $\left(m^{2} \cdot s_{-1}\right)$

$e_{i j}=(i, j)$ component of the deformation rate

$g \quad$ gravitational constant $\left(m \cdot s_{-2}\right)$ $k \quad$ thermal conductivity $\left(W m_{-1} K-1\right)$

$K_{r} \quad$ chemical reaction $\operatorname{rate}(\mathrm{mol} / \mathrm{l} / \mathrm{s})$

$K_{t} \quad$ thermal diffusion $\operatorname{rate}(\mathrm{mol} / \mathrm{l} / \mathrm{s})$

$P_{y} \quad$ yield stress of the fluid()

$T_{\infty} \quad$ free stream temperature $(K)$

$t \quad \operatorname{time}(s)$

$u \quad \operatorname{velocity}(\mathrm{m} / \mathrm{s})$

$v \quad$ constant velocity $(\mathrm{m} / \mathrm{s})$

$\beta \quad$ thermal expansion of fluid

$\beta^{*} \quad$ thermal expansion of concentration

$\rho \quad \operatorname{density}\left(\mathrm{kg} / \mathrm{m}^{3}\right)$

$\nu \quad$ kinematics viscosity $\left(\mathrm{m}^{2} / \mathrm{s}\right)$

\section{REFERENCES}

Ahmed, N., Khan, U., Khan, S.I., Bano, S., and Din, S.T.M., 2017, "Effects on magnetic field in squeezing flow of a Casson fluid between parallel plates," Journal of King Saud University -Science, 29, 119-125. http://dx.doi.org/10.1016/j.jksus.2015.03.006.

Alkasasbeh, H.T., 2017, "Steady state transport phenomena on induced magnetic field modeling for non-Newtonian tangent hyperbolic fluid from an isothermal sphere with Soret and Dufour effect," Frontiers in Heat and Mass Transfer, 9.

http://dx.doi.org/10.5098/hmt.9.17.

Alkasasbeh, H.T., 2018, "Numerical solutions on heat transfer magnetohydrodynamicflow of micropolar Casson fluid over a horizontal circular cylinder with thermal radiation," Frontiers in Heat and Mass Transfer, 10.

http://dx.doi.org/10.5098/hmt.10.32.

Animasaun, I., Adebile, E., and Fagbade, A., 2016, "Casson fluid flow with variable thermo-physical property along exponentially stretching sheet with suction and exponentially decaying internal heat generation using the homotopy analysis method," Journal of the Nigerian Mathematical Societyl, 35, 1-17.

http://dx.doi.org/10.1016/j.jnnms.2015.02.001.

Arora, K.L., and Gupta, P.R., 1972, "Magnetohydrodynamic Flow between Two Rotating Coaxial Cylinders under Radial Magnetic Field," The Physics of Fluids, 15.

http://doi.org/10.1063/1.1694041.

Chamkha, A.J., 2004, "Unsteady MHD convective heat and mass transfer past a semi-infinite vertical permeable moving plate with heat absorption," International Journal of Engineering Science, 42, 217-230. http://dx.doi.org/10.1016/S0020-7225(03)00285-4.

Completo, C., Geraldes, V., and Semiao, V., 2014, "Rheological and dynamical characterization of blood analogue flows in a slit," International Journal of Heat and Fluid Flow, 46, 17-28.

http://dx.doi.org/10.1016/j.ijheatfluidflow.2013.12.008.

Hayat, T., Shehzad, S.A., and A.Alsaedi, 2012, "Soret and Dufour effects on magnetohydrodynamic (MHD) flow of Casson fluid," Applied Mathematics and Mechanics, 33, 1301-1312.

http://dx.doi.org/10.1007/s10483-012-1623-6.

Hayat, T., Rashida, M., Khana, M.I., and Alsaedi, A., 2018, "Melting heat transfer and induced magnetic field effects on flow of water based nanofluid over a rotating disk with variable thickness," Results in Physics, 9, 1618-1630.

http://doi.org/10.1016/j.rinp.2018.04.054. 
Hsua, H.P., Huang, C.J., and Ay, H., 2019, "Influence of MHD on free convection of non Newtonian fluids over a vertical permeable plate in porous media with internal heat generation," Frontiers in Heat and Mass Transfer, 13.

http://dx.doi.org/10.5098/hmt.13.14.

Ibrahim, W., 2016, "The effect of induced magnetic field and convective boundary condition on MHD stagnation point flow and heat transfer of upper-convected Maxwell fluid in the presence of nanoparticle past a stretching sheet," Propulsion and Power Research, 5, 164-175. http://dx.doi.org/10.1016/j.jppr.2016.05.003.

Jafar, K., Nazar, R., Ishak, A., and Pop, I., 2013, "MHD boundary layer flow due to a moving wedge in a parallel streamwith the induced magnetic field," Boundary Value Problems, 20.

http://dx.doi.org/10.1186/1687-2770-2013-20.

Kenjeres, S., 2008, "Numerical analysis of blood flow in realistic arteries subjected to strong non-uniform magnetic fields," International Journal of Heat and Fluid Flow, 29, 752-764.

http://dx.doi.org/10.1016/j.ijheatfluidflow.2008.02.014.

Khalid, A., Khan, I., Khan, A., and Shafie, S., 2015, "Unsteady MHD free convection flow of Casson fluid past over an oscillating vertical plate embedded in a porous medium," Engineering Science and Technology, an International Journal, 18, 309-317.

http://dx.doi.org/10.1016/j.jestch.2014.12.006.

M. Kumari, H.S.T., and Nath, G., 1990, "MHD flow and heat transfer over a stretching surface with prescribed wall temperature or heat flux," Wirme- und Stofffibertragung, 25, 331-336.

Mekheimer, K.S., Saleem, N., Hayat, T., and Hendi, A.A., 2012, "Simultaneous effects of induced magnetic field and heat and mass transfer on the peristaltic motion of second-order fluid in a channel," International journal for numerical methods in fluids, 70, 342-358.

http://dx.doi.org/10.1002/fld.2693.

Mukhapadhyay, S., 2013, "Diffusion of chemically reactive species in Casson fluid flow over an unsteady permeable stretching surface," Journal of Hydrodynamics, 25, 591-598.

http://dx.doi.org/10.1016/S1001-6058(11)60400-X.

Mukhopadhyay, S., De, P.R., Bhattacharyya, K., and Layek, G., 2013, "Casson fluid flow over an unsteady stretching surface," Ain Shams Engi- neering Journal, 4, 933-938.

http://dx.doi.org/10.1016/j.asej.2013.04.004.

Raju, C., Sandeep, N., and Saleem, S., 2016, "Effects of induced magnetic field and homogeneous-heterogeneous reactions on stagnation flow of a Casson fluid," Engineering Science and Technology, an International Journa, 19, 875-887.

http://dx.doi.org/10.1016/j.jestch.2015.12.004.

Reddy, G.J., Raju, R.S., and Rao, J.A., 2018a, "Influence of viscous dissipation on unsteady MHD natural convective flow of Casson fluid over an oscillating vertical plate via FEM," Ain Shams Engineering Journa, 9, 1907-1915.

http://doi.org/10.1016/j.ijheatmasstransfer.2018.08.022.

Reddy, P.C., Raju, M., Reddy, S.H., and Raju, G., 2018b, "Casson Fluid Flow Over A Vertical Porous Plate Under The Existence Of Cross Diffusion Effects In Conducting Field," International Journal of Advanced Scientific Research and Management, $\mathbf{3}$.

Shateyi, S., Mabood, F., and Lorenzini, G., 2017, "Casson Fluid Flow: Free Convective Heat and Mass Transfer over an Unsteady Permeable Stretching Surface Considering Viscous Dissipation," Journal of Engineering Thermophysics, 26, 39-52. http://dx.doi.org/10.1134/S1810232817010052.

Siddiqa, S., Begum, N., Ouazzi, A., Hossain, M.A., and Gorla, R.S.R., 2018, "Heat transfer analysis of Casson dusty fluid flow along a vertical wavy cone with radiating surface," International Journal of Heat and Mass Transfer, 127, 589-596.

http://doi.org/10.1016/j.ijheatmasstransfer.2018.08.022.

Sulochana, C., Ashwinkumar, G., and Sandeep, N., 2016, "Similarity solution of 3D Casson nanofluid flow over a stretching sheet with convective boundary conditions," Journal of King Saud University-Science, 35, 128-141.

http://dx.doi.org/10.1016/j.jnnms.2016.01.001.

Ullah, I., Shafie, S., and Khan, I., 2017, "Effects of slip condition and Newtonian heating on MHD flow of Casson fluid over a nonlinearly stretching sheet saturated in a porous medium," Journal of King Saud University-Science, 29, 250-259.

http://dx.doi.org/10.1016/j.jksus.2016.05.003. 